\title{
Thromboserezidivprophylaxe
}

\section{Zulassungserweiterungen für Dalteparin}

Das niedermolekulare Heparin Dalteparin wurde vor Kurzem in zwei neuen Indikationen zugelassen: Zur Therapie der akuten tiefen Venenthrombose und der Lungenembolie sowie zur Rezidivprophylaxe venöser Thromboembolien (VTE) bei onkologischen Patienten.

Für die Thromboserezidivprophylaxe bei Tumorpatienten bestand bislang für keines der am Markt verfügbaren niedermolekularen Heparine eine Zulassung. „Weil niedermolekulare Heparine aber in der aktuellen S2-Leitlinie zur Diagnostik und Therapie der VTE und der Lungenembolie außer für die Initialtherapie auch bereits für die Sekundärprophylaxe empfohlen wurden, befanden sich die Therapeuten immer in einem gewissen Dilemma“, erläuterte Hanno Riess, Berlin. „Mit der Indikationserweiterung für Dalteparin wurde deshalb eine bedeutende therapeutische Lücke geschlossen", so Riess.

VTE sind eine häufige und oft unterdiagnostizierte Komplikation bei malignen Erkrankungen. Zudem ist das Rezidivrisiko hoch. Um diese Gefahr zu senken, müssen Tumorpatienten mindestens bis zur Remission, bei entsprechendem Befund lebenslang, eine Antikoagulationstherapie erhalten. „Die Rezidivprophylaxe ist auch deshalb extrem wichtig, weil VTE einen maßgeblichen Einfluss auf den Therapieerfolg und die Prognose der Krebspatienten haben", ergänzte Riess.

\section{Risikoreduktion für Rezidivthrombosen}

Dass Dalteparin (Fragmin ${ }^{\circledast}$ ) als Medikament zur VTE-Rezidivprophylaxe dem Vitamin-K-Antagonisten Warfarin in der Wirksamkeit eindeutig überlegen ist, konnte in der prospektiven, offenen Studie CLOT* gezeigt werden (Lee AY et al., 2003, N Eng J Med

\section{CLOT-Studie: Endpunkte}

\begin{tabular}{|lll|}
\hline $\begin{array}{l}\text { Dalteparin } \\
(\mathrm{n}=336)\end{array}$ & $\begin{array}{l}\text { Warfarin } \\
(\mathrm{n}=336)\end{array}$ \\
\hline $\begin{array}{l}\text { venöse Thromboembolien } \\
\text { tiefe Venenthrombosen } \\
\text { (ohne Lungenembolien) }\end{array}$ & $27(9 \%)$ & $53(17 \%)$ \\
\hline $\begin{array}{l}\text { Lungenembolien } \\
\text { tödliche Lungenembolien }\end{array}$ & 14 & 37 \\
\hline Blutungen & 13 & 16 \\
\hline $\begin{array}{l}\text { größere Blutungen } \\
\text { Sterblichkeit }\end{array}$ & $57(14 \%)$ & $74(19 \%)$ \\
\hline nach Lee AY et al., 2003, N Eng JMed 349: 146-153 & $19(6 \%)$ & $12(4 \%)$ \\
\hline
\end{tabular}

349: 146-153). Innerhalb eines halben Jahres erlitten 53 Patienten unter Warfarin, aber nur 27 Patienten unter Dalteparin eine Rezidivthrombose (Tab.). Damit war das Risiko unter Dalteparin um mehr als die Hälfte niedriger als unter der Therapie mit dem Vitamin-K-Antagonisten (Hazard-Ratio 0,48; $p=0,002$ ). „Durch die Zulassungserweiterung für Dalteparin, die sich auf diese Studienergebnisse stützt, ist ein seit Langem in der klinischen Praxis bewährtes niedermolekulares Heparin jetzt noch breiter einsetzbar geworden", fasste Riess abschließend zusammen. SW

* Randomized Comparison of Low Molecular Weight Heparin versus Oral Anticoagulant Therapy for Long Term Anticoagulation in Cancer Patients with Venous Thromboembolism Quelle: Veranstaltung der Pfizer Pharma GmbH

\section{Tuberöse Sklerose - subependymales Riesenzellastrozytom \\ RAD001-Behandlung für TSC-Patienten mit SEGA}

Die Zulassung für den oral verfügbaren mTOR-Inhibitor RAD001 zur Behandlung von Patienten mit tuberöser Sklerose (TSC), assoziiert mit subependymalem Riesenzellastrozytom (SEGA), wurde im Juli 2010 bei der European Medicines Agency (EMA) beantragt. Im Rahmen eines Expanded Access Program (EAP) steht den Betroffenen RAD001 bereits vorab bis zur Zulassung zur Verfügung.

In einer einarmigen Phase-II-Studie mit Patienten mit TSC-assoziierten SEGA konnte unter RAD001 das SEGA-Volumen bei 75\% der Patienten nach sechs Monaten Therapiedauer um mindestens $30 \%$ signifikant reduziert werden ( $p<0,001$; Krueger DA et al., 2010, N Engl J Med 363: 1801-1811). Gleichzeitig ging die Häufigkeit epileptischer Krampfanfälle signifikant zurück $(p=0,02)$, und die Verträglichkeit von RAD001 wurde insgesamt als gut bewertet. Auf Basis dieser Daten wurde die Zulassung bei der EMA beantragt.

\section{Expanded Access Program}

Um Patienten mit TSC-assoziierten SEGA bereits vorab den Zugang zu einer Behandlung mit RAD001 zu ermöglichen, wurde vor dem Hintergrund des dringenden medizinischen Bedarfs die EAP-Studie CRAD001MIC02 konzipiert. Primärer Endpunkt der offenen, multizentrischen erweiterten Zugangsstudie sind die Sicherheit und Verträglichkeit von RAD001. Sekundärer Endpunkt ist das beste Gesamtansprechen, das durch den behandelnden Prüfarzt definiert wird. Die EAP-Stu- die begann im März und läuft in Deutschland etwa bis Herbst dieses Jahres. Die Studienleitung hat Olaf Witt, Deutsches Krebsforschungszentrum Heidelberg, inne. Eingeschlossen werden können Patienten in einem Alter ab drei Jahren mit gesicherter Diagnose von TSC und mindestens einem über CT/MRT identifizierten SEGA. Mehr Informationen zurEAP-Studie CRAD001MIC02 erhalten Sie unter 01802 - 232300 beim Novartis Info-Service.

Quelle: Information der Novartis Pharma GmbH 